\title{
Editorial
}

Cerebrovascular Diseases
Cerebrovasc Dis 2020;49:121-123

DOI: 10.1159/000506680
Received: February 8, 2020

Accepted: February 17, 2020

Published online: March 20, 2020

\section{Avoiding Stroke: A Continuous Monitoring Challenge}

\author{
Jakub Gumprecht ${ }^{\mathrm{a}, \mathrm{c}}$ Magdalena Domek ${ }^{\mathrm{a}, \mathrm{d}}$ Andrew M. Hilla, e \\ Gregory Y.H. Lip ${ }^{a-c}$ \\ a Liverpool Centre for Cardiovascular Science, University of Liverpool and Liverpool Heart and Chest Hospital, \\ Liverpool, UK; ${ }^{b}$ Aalborg Thrombosis Research Unit, Department of Clinical Medicine, Aalborg University, Aalborg, \\ Denmark; ' ${ }^{\circ}$ epartment of Cardiology, Congenital Heart Diseases and Electrotherapy, Medical University of Silesia, \\ Silesian Centre for Heart Diseases, Zabrze, Poland; ' Department of Internal Diseases, Diabetology and Nephrology, \\ Medical University of Silesia, Zabrze, Poland; ' St. Helens and Knowsley Teaching Hospitals, Whiston, UK
}

Stroke is a leading cause of death and disability worldwide. Risk factors such as advancing age, hypertension, cigarette smoking, atrial fibrillation (AF), diabetes mellitus and prior cardiovascular disease $[1,2]$ are well established and provide significant opportunities through public health policy to reduce both the disease burden and the socio-economic cost of stroke care [3]. In approximately $30 \%$ of cases, the aetiology is undetermined despite standard investigations [4]. A proportion of these cryptogenic events will be induced by paroxysmal, "silent" AF. However, establishing the relative burden of silent paroxysmal AF upon stroke risk has been challenging, particularly as continuous cardiac monitoring has developed faster than the ability to contextualise the findings upon stroke risk [5]. The minimum duration of arrhythmia detected on prolonged monitoring considered clinically significant enough to influence ischemic stroke risk is unclear. Current American Heart Association guidelines recommend a threshold of $30 \mathrm{~s}$ for diagnosis of AF, with attention to temporal AF burden aggregation: the concept that a patient with a small number of prolonged episodes of AF has a higher AF density than one with many brief episodes [6].

Premature atrial contractions (PAC), previously thought to be benign, may also increase ischemic stroke risk. Several studies have shown the relationship between excessive PACs and an increased risk of ischemic stroke
[7-9]. A systematic review and meta-analysis based on 11 studies revealed that frequent PACs were associated with a significantly raised risk of stroke and mortality (risk ratio $1.41,95 \%$ CI $1.25-1.60$; risk ratio 2,17 , 95\% CI $1.13-$ 1.41 , respectively) [10]. In line with those results, the findings from a UK case-control study involving 461 patients with ischemic stroke or transient ischemic attack (TIA) also revealed an independent association of excessive PACs burden and ischemic events among individuals without prior AF history [7]. After adjusting the analysis for other common risk factors, the excessive number of PACs, defined as $\geq 200 \mathrm{PACs} / 24 \mathrm{~h}$, proved to be the superior risk factor for the cryptogenic stroke subtype (OR 1.95; 95\% CI 1.16-3.28) [7]. One possible rationale might be the presence of an atrial myopathy with the electroanatomical changes of the left atrium influencing slower conduction velocities or lower atrial voltages [7].

Although the association between PACs and stroke prevalence has been proven, selecting the optimal method of arrhythmia detection is challenging. Traditional clinical practice of $24 \mathrm{~h}$ ECG monitoring after stroke or TIA has a low detection rate; increasing this to $72 \mathrm{~h}$ or 7 days improves this but still has low sensitivity for shorter episodes of paroxysmal AF or PACs. Prolonged monitoring using standard Holter-ECG devices is likely to be poorly tolerated, causing skin irritations and being strictly dependent

\section{KARGER}

(C) 2020 S. Karger AG, Basel

karger@karger.com

www.karger.com/ced
Prof. Gregory Y.H. Lip

Liverpool Centre for Cardiovascular Science

University of Liverpool and Liverpool Heart and Chest Hospital

Liverpool L7 8TX (UK)

E-Mail gregory.lip@liverpool.ac.uk 
on patients' compliance [12]. Hence, long-term non-invasive monitoring may provide a solution to the problem.

In this issue of the journal, Todo et al. [13] demonstrate an association of excessive PACs detected by the implantable cardiac monitor (ICM) with the new-onset AF following cryptogenic stroke among 66 Japanese patients. The analysis also indicated that in patients with frequent PACs, the time between the ischemic event and establishment of an AF diagnosis was substantially lower. Hence, if we look longer, look harder and with more sophisticated ways, our detection of AF will be correspondingly increased.

The CRYSTAL-AF study, which compared the standard approach of $24 \mathrm{~h}$ Holter-ECG monitoring versus ICM after cryptogenic stroke, also confirmed the significant superiority of ICM-arm in AF detection (30 vs. 3.0\% in 36 months), emphasizing the need for long-term monitoring $[14,15]$. This type of implantable heart electrical activity monitor provides the opportunity of thorough ECG analysis of automatically detected arrhythmias or those indicated by the patients themselves. Thus, an ICM may constitute an accurate and effective diagnostic tool in patients at high risk of cryptogenic stroke [16].

Also, ICM implantation is an invasive procedure carrying the risk of complications. Therefore, it should be considered in patients where the clinical suspicion of AF is high, non-invasive approaches have been considered and a comprehensive risk-benefit ratio assessment has been undertaken.

By comparison, wearable devices (including bands, smartwatches or smartphone apps) offer non-invasive long-term cardiac monitoring and are consumer-purchased rather than medically directed devices. Studies of wearables by Apple [17] and Huawei [18] into their wearable devices demonstrate positive predictive values of 0.84 and 0.91 , respectively, for detecting AF when used in conjunction with ECG patches. However, their sensitivity across an asymptomatic population is low, and detection of more complex arrhythmias is limited. Whilst these offer promising approaches for the future, the threshold by which these could be considered medical devices has not yet been reached.

Various types of wearable external loop recorders in the form of jackets, vests or belts have also been developed $[8,19]$. They allow a non-invasive prolonged assessment of at least two ECG recording leads, which considerably improves the arrhythmia detection efficiency. A subanalysis from EMBRACE trial revealed the increase in AF detection rate up to $40 \%$ in individuals with $\geq 1,500 / 24 \mathrm{~h}$ PACs during 90 days of cardiac tracking using the ECGbelt compared to standard care [8].
According to the AF-SCREEN International Collaboration, a pathway that combines the current routine-care approach of $24 \mathrm{~h}$ monitoring and intensive ECG monitoring for at least $72 \mathrm{~h}$ allows detecting the new-onset $\mathrm{AF}$ in around $25 \%$ of patients with cryptogenic stroke [20]. The timespan might then be extended up to 90 days of monitoring using wearable non-invasive devices in patients without documented arrhythmia $[8,20]$.

In patients where ischemic stroke or TIA has already occurred, the treating clinician must balance the probability of cardiac arrhythmia being the cause of the underlying event, taking into account the stroke subtype, and utilizing predictive tools such as the $\mathrm{C}_{2} \mathrm{HEST}$ score when considering the best strategy for detection of arrhythmia and the significance of any findings [21]. It is worth noting that there is currently no evidence supporting anticoagulation therapy in patients with cryptogenic stroke but without ECG-documented arrhythmia nor with atrial myopathy only [11]. Prolonged monitoring aiming at an increase in the rate of AF detection seems to be a comprehensive and holistic approach to improve stroke prevention.

\section{Acknowledgement}

Dr. Jakub Gumprecht was supported by the Polish Cardiac Society Club 30 Specialized Research Fellowship Grant for Early Career Researchers.

\section{Statement of Ethics}

Ethics approval was not required for the purpose of the editorial.

\section{Disclosure Statement}

None directly related to this paper. G.Y.H.L. consultant for Bayer/Janssen, BMS/Pfizer, Medtronic, Boehringer Ingelheim, Novartis, Verseon and Daiichi-Sankyo. Speaker for Bayer, BMS/ Pfizer, Medtronic, Boehringer Ingelheim, and Daiichi-Sankyo. No fees are directly received personally. Other authors have no conflict of interest.

\section{Funding Sources}

The manuscript was not founded.

\section{Author Contributions}

All co-authors had an equal contribution to the manuscript. 


\section{References}

1 Guo Y, Wang H, Tian Y, Wang Y, Lip GY. Multiple risk factors and ischaemic stroke in the elderly Asian population with and without atrial fibrillation. An analysis of 425,600 Chinese individuals without prior stroke. Thromb Haemost. 2016 Jan;115(1): 184-92.

2 Wolf PA, D'Agostino RB, Belanger AJ, Kannel WB. Probability of stroke: a risk profile from the Framingham Study. Stroke. 1991 Mar;22(3):312-8.

3 Public Health England. The Size of the Prize in Cardiovascular Disease (CVD) Prevention: England. [Accessed Feb 1, 2020]. Available from: https://www.healthcheck.nhs.uk/ seecmsfile/?id=983.

4 Benjamin EJ, Blaha MJ, Chiuve SE, Cushman M, Das SR, Deo R, et al.; American Heart Association Statistics Committee and Stroke Statistics Subcommittee. Heart Disease and Stroke Statistics-2017 Update: A Report From the American Heart Association. Circulation. 2017 Mar;135(10):e146-603.

5 Passman R, Bernstein RA. New Appraisal of Atrial Fibrillation Burden and Stroke Prevention. Stroke. 2016 Feb;47(2):570-6.

6 Chen LY, Chung MK, Allen LA, Ezekowitz M, Furie KL, McCabe P, et al. Atrial Fibrillation Burden: Moving Beyond Atrial Fibrillation as a Binary Entity: A Scientific Statement From the American Heart Association. Circulation. 2018 May;137(20):e623-44.

7 Sajeev JK, Koshy AN, Dewey H, Kalman JM, Rajakariar K, Tan MC, et al. Association between excessive premature atrial complexes and cryptogenic stroke: results of a casecontrol study. BMJ Open. 2019 Jul;9(7): e029164.
8 Gladstone DJ, Dorian P, Spring M, Panzov V, Mamdani M, Healey JS, et al.; EMBRACE Steering Committee and Investigators. Atrial premature beats predict atrial fibrillation in cryptogenic stroke: results from the EMBRACE trial. Stroke. 2015 Apr;46(4):936-41.

9 Vinther KH, Tveskov C, Möller S, Auscher S, Osmanagic A, Egstrup K. Excessive Premature Atrial Complexes and the Risk of Recurrent Stroke or Death in an Ischemic Stroke Population. J Stroke Cerebrovasc Dis. 2017 Jun;26(6):1163-70.

10 Huang BT, Huang FY, Peng Y, Liao YB, Chen $\mathrm{F}$, Xia TL, et al. Relation of premature atrial complexes with stroke and death: systematic review and meta-analysis. Clin Cardiol. 2017 Nov;40(11):962-9.

11 Lip G, Freedman B, De Caterina R, Potpara TS. Stroke prevention in atrial fibrillation: Past, present and future. Comparing the guidelines and practical decision-making. Thromb Haemost. 2017 Jun;117(7):1230-9.

12 Tu HT, Spence S, Kalman JM, Davis SM. Twenty-eight day Holter monitoring is poorly tolerated and insensitive for paroxysmal atrial fibrillation detection in cryptogenic stroke. Intern Med J. 2014 May;44(5):505-8.

13 Todo K, Iwata T, Doijiri R, Yamagami H, Morimoto M, Hashimoto T, et al. Frequent premature atrial contractions in cryptogenic stroke predict atrial fibrillation detection with insertable cardiac monitoring. Cerebrovasc Dis. 2020, Epub ahead of print.

14 Sanna T, Diener HC, Passman RS, Di Lazzaro V, Bernstein RA, Morillo CA, et al.; CRYSTAL AF Investigators. Cryptogenic stroke and underlying atrial fibrillation. $\mathrm{N}$ Engl J Med. 2014 Jun;370(26):2478-86.
15 Thijs VN, Brachmann J, Morillo CA, Passman RS, Sanna T, Bernstein RA, et al. Predictors for atrial fibrillation detection after cryptogenic stroke: results from CRYSTAL AF. Neurology. 2016 Jan;86(3):261-9.

16 Israel C, Kitsiou A, Kalyani M, Deelawar S, Ejangue LE, Rogalewski A, et al. Detection of atrial fibrillation in patients with embolic stroke of undetermined source by prolonged monitoring with implantable loop recorders. Thromb Haemost. 2017 Oct;117(10):1962-9.

17 Perez MV, Mahaffey KW, Hedlin H, Rumsfeld JS, Garcia A, Ferris T, et al.; Apple Heart Study Investigators. Large-scale assessment of a smartwatch to identify atrial fibrillation. N Engl J Med. 2019 Nov;381(20):1909-17.

18 Guo Y, Wang H, Zhang H, Liu T, Liang Z, Xia $\mathrm{Y}$, et al.; MAFA II Investigators. Mobile Photoplethysmographic Technology to Detect Atrial Fibrillation. J Am Coll Cardiol. 2019 Nov;74(19):2365-75.

19 Kalarus Z, Balsam P, Bandosz P, Grodzicki T, Kaźmierczak J, Kiedrowicz R, et al. NOninvasive monitoring for early detection of Atrial fibrillation: rationale and design of the NOMEDAF study. Kardiol Pol. 2018;76(10):1482-5.

20 Schnabel RB, Haeusler KG, Healey JS, Freedman B, Boriani G, Brachmann J, et al. Searching for Atrial Fibrillation Poststroke: A White Paper of the AF-SCREEN International Collaboration. Circulation. 2019 Nov;140(22): 1834-50.

21 Li YG, Bisson A, Bodin A, Herbert J, Grammatico-Guillon L, Joung B, et al. C2 HEST Score and Prediction of Incident Atrial Fibrillation in Poststroke Patients: A French Nationwide Study. J Am Heart Assoc. 2019 Jul; 8(13):e012546. 